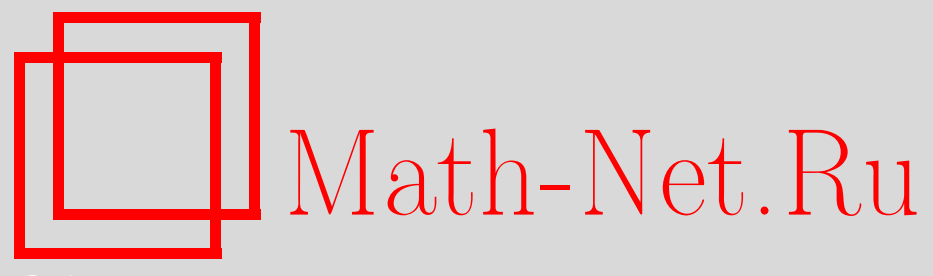

А. А. Бирюков, М. А. Шлеенков, Представление вероятностей квантовых переходов функциональным интегралом в пространстве энергетических состояний, Вестн. Сам. гос. техн. ун-та. Сер. Физ.мат. науки, 2015, номер 2, 221-240

DOI: https://doi.org/10.14498/vsgtu1392

Использование Общероссийского математического портала MathNet.Ru подразумевает, что вы прочитали и согласны с пользовательским соглашением

http: //www . mathnet.ru/rus/agreement

Параметры загрузки:

IP : 3.82 .47 .9

26 апреля 2023 г., 10:41:52

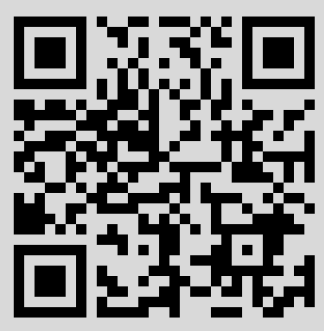




\title{
ПРЕДСТАВЛЕНИЕ ВЕРОЯТНОСТЕЙ КВАНТОВЫХ ПЕРЕХОДОВ ФУНКЦИОНАЛЬНЫМ ИНТЕГРАЛОМ В ПРОСТРАНСТВЕ ЭНЕРГЕТИЧЕСКИХ СОСТОЯНИЙ*
}

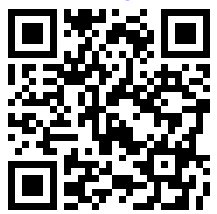

\section{А. А. Бирюков, М. А. Шлеенков}

Самарский государственный университет,

Россия, 443011, Самара, ул. Академика Павлова, 1.

\begin{abstract}
Аннотация
В рамках формализма функционального интегрирования (интегрирования по траекториям) излагается непертурбативный метод описания динамики многоуровневых квантовых систем (атомов, молекул, наносистем), взаимодействующих с полем лазерного излучения высокой интенсивности. Вероятности переходов между состояниями квантовой системы под действием внешнего электромагнитного поля представляются функциональным интегралом в энергетическом представлении (в пространстве энергетических состояний исследуемой многоуровневой квантовой системы). На основании предложенного метода вычисляются вероятности переходов двухатомных молекул между энергетическими уровнями вращательных степеней свободы под действием серии пикосекундных импульсов лазерного излучения. Проведено численное моделирование динамики населенностей различных вращательных квантовых состояний молекул ${ }^{14} \mathrm{~N}_{2}$ и ${ }^{15} \mathrm{~N}_{2}$ под действием серии пикосекундых лазерных импульсов различной интенсивности и с различным периодом группы импульсов. Показано, что при определенных интервалах между лазерными импульсами наблюдается явление резонанса переноса населенности с низших на более высокие вращательные уровни исследуемых молекул. Данный резонанс исследуется при различных интенсивностях лазерных импульсов. Примечательно, что параметры лазерного излучения, при которых наблюдается резонансный переход населенностей молекул, различны для ${ }^{14} \mathrm{~N}_{2}$ и ${ }^{15} \mathrm{~N}_{2}$. Полученные результаты указывают на возможность селективного возбуждения изотопов под действием групп ультракоротких лазерных импульсов путем варьирования их интенсивности и периода следования в группе. Результаты численного моделирования количественно согласуются с результатами экспериментальных исследований [Phys. Rev. Lett., 2012, vol. 109, 043003].
\end{abstract}

(C) 2015 Самарский государственный технический университет.

\section{Образец для цитирования}

Бирюков А. А., Шлеенков М. А. Представление вероятностей квантовых переходов функциональным интегралом в пространстве энергетических состояний // Вестн. Сам. гос. техн. ун-та. Сер. Физ.-мат. науки, 2015. Т. 19, № 2. С. 221-240. doi: $10.14498 /$ vsgtu1392.

\section{Сведения об авторах}

Александр Александрович Бирюков (к.ф.-м.н., проф.; biryukov@samsu.ru), зав. кафедрой, каф. общей и теоретической физики.

Марк Александрович Шлеенков (к.ф.-м.н.; shleenkov@list.ru; автор, ведущий переписку), ассистент, каф. общей и теоретической физики.

*Настоящая статья представляет собой расширенный вариант доклада [1], сделанного авторами на Четвёртой международной конференции «Математическая физика и её приложения» (Россия, Самара, 25 августа - 1 сентября 2014). 
Ключевые слова: интегралы по траекториям, многофотонные процессы, нерезонансные процессы, энергетическое представление, молекулы азота, ультракороткие лазерные импульсы, нелинейное взаимодействие, селективное возбуждение вращательных состояний.

doi: http://dx.doi.org/10.14498/vsgtu1392

Введение. В настоящее время активно изучаются нелинейные процессы взаимодействия наносистем с интенсивным электромагнитным полем [2-4], возбуждение и диссоциация различных молекул [5-7], ионизация атомов под действием лазерного излучения [8-10], многофотонный фотоэффект [11, 12] и др. В работах [13-15] было показано, что под действием нерезонансного электромагнитного излучения, состоящего из последовательности лазерных импульсов, наблюдается заселение высоковозбужденных вращательных состояний молекул азота ${ }^{14} \mathrm{~N}_{2}$ и ${ }^{15} \mathrm{~N}_{2}$. При определенной периодичности лазерных импульсов в группе наблюдается резонансное увеличение вероятности заселения высоких вращательных состояний облучаемой молекулы. Вращательная динамика молекулы в данном эксперименте может быть описана в рамках рамановских переходов.

Под действием интенсивных полей помимо однофотонных процессов высокую вероятность имеют многофотонные процессы, для которых требуется особый подход при построении математических моделей. Для описания процессов нелинейной оптики разработаны разные теоретические методы, базирующиеся на интегральном уравнении для $S$-матрицы, на нестационарном уравнении Шрёдингера, использующие фейнмановский подход к теории возмущений.

В настоящее время представляется актуальным получить уравнения, описывающие динамику квантовых систем в сильных электромагнитных полях в рамках непертурбативного подхода. Одним из таких подходов является формализм функционального интегрирования, предложенного Р. Фейнманом [16].

В данной работе вероятности квантовых переходов многоуровневой системы, взаимодействующей с электромагнитным полем, записываются в виде интеграла по траекториям от действительного функционала в энергетическом представлении.

В рамках предложенного подхода $[17,18]$ описывается многофотонное возбуждение вращательных степеней свободы неполярной двухатомной молекулы азота ${ }^{14} \mathrm{~N}_{2}$ при воздействии групп ультракоротких лазерных импульсов с различными периодами следования и максимальными значениями интенсивности.

1. Описание эволюции квантовой системы. Представление матрицы плотности функциональным интегралом в энергетическом представлении. Рассмотрим многоуровневую квантовую систему, взаимодействующую с электромагнитным полем, которая описывается гамильтнианом

$$
\hat{H}=\hat{H}_{\text {syst }}+\hat{V}_{\text {inf }}(t)
$$

где

$$
\hat{H}_{\text {syst }}=\sum_{l} E_{l}|l\rangle\langle l|
$$


- гамильтониан квантовой системы; $E_{l}$ - значения энергий квантовой системы в стационарных состояниях $|l\rangle$; вектора $|l\rangle$ ортонормированны $\left(\left\langle l^{\prime} \mid l\right\rangle=\delta_{l^{\prime} l}\right)$ и образуют полную систему:

$$
\begin{gathered}
\sum_{l}|l\rangle\langle l|=1 \\
\hat{V}_{\text {inf }}(\tau)=e E_{0} \cos (\Omega \tau) \sum_{l^{\prime}, l}\left|l^{\prime}\right\rangle\left\langle l^{\prime}|\hat{x}| l\right\rangle\langle l|
\end{gathered}
$$

- оператор взаимодействия квантовой системы с электромагнитным полем с частотой $\Omega$, амплитудой $E_{0} ; e-$ заряд частицы; $\hat{x}$ - оператор координаты частицы [19].

Описание эволюции многоуровневой квантовой системы проведем в представлении взаимодействия. Уравнение эволюции статистического оператора имеет вид

$$
\hat{\rho}(t)=\hat{U}_{D}(t) \hat{\rho}(0) \hat{U}_{D}^{+}(t) .
$$

В выражении (1) оператор эволюции имеет вид

$$
\hat{U}_{D}\left(t, t_{0}\right)=T \exp \left[-\frac{\imath}{\hbar} \int_{t_{0}}^{t} \hat{V}_{D}(\tau) d \tau\right],
$$

где

$$
\hat{V}_{D}(\tau)=\exp \left[\frac{\imath}{\hbar} \hat{H}_{\text {syst }} \tau\right] \hat{V}_{\text {inf }}(\tau) \exp \left[-\frac{\imath}{\hbar} \hat{H}_{\text {syst }} \tau\right]
$$

Ради упрощения дальнейших выражений представим (2) в виде

$$
\hat{V}_{\mathrm{inf}}(\tau)=\sum_{l^{\prime}, l} \hbar \Omega_{l^{\prime} l}^{R} \cos (\Omega \tau)\left|l^{\prime}\right\rangle\langle l|,
$$

где

$$
\Omega_{l^{\prime} l}^{R}=\frac{q x_{l^{\prime} l} E_{0}}{\hbar}
$$

— частота Раби; $x_{l^{\prime} l}=\left|\left\langle l^{\prime}|\hat{x}| l\right\rangle\right|$ - абсолютная величина матричного элемента оператора координаты.

Используя выражения (3), (4), оператор взаимодействия (2) представим в виде, который удобен для конкретизации оператора эволюции:

$$
\hat{V}_{D}(\tau)=\sum_{l^{\prime}, l} \hbar \Omega_{l^{\prime} l}^{R} \cos (\Omega \tau) \exp \left[\imath \omega_{l^{\prime} l} \tau\right]\left|l^{\prime}\right\rangle\langle l|,
$$

где $\omega_{l^{\prime} l}$ - частота квантового перехода системы между стационарными состояниями с энергиями $E_{l^{\prime}}$ и $E_{l}$ :

$$
\omega_{l^{\prime} l}=\frac{E_{l^{\prime}}-E_{l}}{\hbar}
$$

Используя формулы Эйлера для представления тригонометрических функций комбинациями комплексных экспонент, представим (5) в виде

$$
\sum_{l^{\prime}, l} X_{l^{\prime}, l}\left|l^{\prime}\right\rangle\langle l|
$$


где

$$
X_{l^{\prime} l}(\tau)=\frac{1}{2} \hbar \Omega_{l^{\prime} l}^{R}\left(\exp \left[-\imath\left(\Omega-\omega_{l^{\prime}, l}\right) \tau\right]+\exp \left[\imath\left(\Omega+\omega_{l^{\prime}, l}\right) \tau\right]\right) .
$$

Запишем уравнение эволюции статистической матрицы плотности (1) в энергетическом представлении:

$$
\rho_{n_{f} m_{f}}(t)=\sum_{n_{i n}, m_{i n}}\left\langle n_{f}\left|\hat{U}_{D}(t)\right| n_{0}\right\rangle \rho_{n_{0}, m_{0}}\left\langle m_{0}\left|\hat{U}_{D}^{+}(t)\right| m_{f}\right\rangle
$$

где

$$
\rho_{n_{f} m_{f}}(t)=\left\langle n_{f}|\hat{\rho}(t)| m_{f}\right\rangle, \quad \rho_{n_{0}, m_{0}}=\left\langle n_{0}|\hat{\rho}(0)| m_{0}\right\rangle .
$$

Вектора $|n\rangle,|m\rangle$ являются собственными векторами гамильтониана системы $H_{\text {syst. }}$

Ядро оператора эволюции $\left\langle n_{f}\left|\hat{U}_{D}(t, 0)\right| n_{0}\right\rangle$ представим в виде произведения элементарных ядер, используя групповые свойства оператора $\hat{U}_{D}$ и полноту векторов-состояний $\left|n_{k}\right\rangle$ :

$$
\left\langle n_{f}\left|\hat{U}_{D}(t, 0)\right| n_{0}\right\rangle=\sum_{n_{1}, \ldots, n_{K}=1}^{N} \prod_{k=1}^{K+1}\left\langle n_{k}\left|\hat{U}_{D}\left(t_{k}, t_{k-1}\right)\right| n_{k-1}\right\rangle
$$

где $t_{K+1}=t, n_{K+1}=n_{f}, t_{0}=0$ и

$$
\hat{U}_{D}\left(t_{k}, t_{k-1}\right)=\exp \left[-\frac{\imath}{\hbar} \int_{t_{k-1}}^{t_{k}} \hat{V}_{D}(\tau) d \tau\right]
$$

где $t_{k}>t_{k-1}$.

Докажем, что для малых интервалов времени $\left(\left(t_{k}-t_{k-1}\right) \rightarrow 0\right)$ ядро оператора эволюции $\left\langle n_{k}\left|\hat{U}_{D}\left(t_{k}, t_{k-1}\right)\right| n_{k-1}\right\rangle$ имеет вид

$$
\left\langle n_{k}\left|\hat{U}_{D}\left(t_{k}, t_{k-1}\right)\right| n_{k-1}\right\rangle=\int_{0}^{1} \exp \left[-\imath S\left[n_{k}, t_{k} ; n_{k-1}, t_{k-1} ; \xi_{k-1}\right]\right] d \xi_{k-1}
$$

где

$$
\begin{aligned}
S\left[n_{k}, t_{k} ; n_{k-1}, t_{k-1} ; \xi_{k-1}\right]=2 \pi\left(n_{k}-n_{k-1}\right) \xi_{k-1}+ \\
+\Omega_{n_{k} n_{k-1}}^{R}\left(\cos \left(2 \pi\left(n_{k}-n_{k-1}\right) \xi_{k-1}+\left(\Omega+\omega_{n_{k}, n_{k-1}}\right) \frac{t_{k}+t_{k-1}}{2}\right)+\right. \\
\left.\quad+\cos \left(2 \pi\left(n_{k}-n_{k-1}\right) \xi_{k-1}-\left(\Omega-\omega_{n_{k}, n_{k-1}}\right) \frac{t_{k}+t_{k-1}}{2}\right)\right)\left(t_{k}-t_{k-1}\right)
\end{aligned}
$$

- безразмерное действие (в единицах $\hbar$ ) в энергетическом представлении.

Для доказательства справедливости выражений $(7),(8)$ для ядра оператора эволюции, используя (6), представим $\left\langle n_{k}\left|\hat{U}_{D}\left(t_{k}, t_{k-1}\right)\right| n_{k-1}\right\rangle$ для малых интервалов времени $\left(\left(t_{k}-t_{k-1}\right) \rightarrow 0\right)$ в виде

$$
\left\langle n_{k}\left|\hat{U}_{D}\left(t_{k}, t_{k-1}\right)\right| n_{k-1}\right\rangle=\left\langle n_{k} \mid n_{k-1}\right\rangle-\frac{\imath}{\hbar} \int_{t_{k-1}}^{t_{k}}\left\langle n_{k}\left|\hat{V}_{D}(\tau)\right| n_{k-1}\right\rangle d \tau
$$


Докажем, что

$$
\begin{aligned}
& \left\langle n_{k}\left|\hat{V}_{D}(\tau)\right| n_{k-1}\right\rangle=\int_{0}^{1} \exp \left[-2 \pi \imath\left(n_{k}-n_{k-1}\right) \xi_{k-1}\right] \times \\
& \times \hbar \Omega_{n_{k} n_{k-1}}^{R}\left(\cos \left(2 \pi \xi_{k-1}\left(n_{k}-n_{k-1}\right)-\left(\Omega-\omega_{n_{k} n_{k-1}}\right) \tau\right)+\right. \\
& \left.\quad+\cos \left(2 \pi \xi_{k-1}\left(n_{k}-n_{k-1}\right)+\left(\Omega+\omega_{n_{k} n_{k-1}}\right) \tau\right)\right) d \xi_{k-1} .
\end{aligned}
$$

Для доказательства (10) построим матричный элемент взаимодействия

$$
\left\langle n_{k}\left|\hat{V}_{D}(\tau)\right| n_{k-1}\right\rangle=\hbar \sum_{l^{\prime}, l} X_{l^{\prime}, l} \delta_{n_{k} l^{\prime}} \delta_{\left(n_{k}-n_{k-1}\right),\left(l^{\prime}-l\right)} \delta_{l n_{k-1}},
$$

где была использована ортонормированность векторов-состояний квантовой системы:

$$
\delta_{n_{k} l^{\prime}}=\left\langle n_{k} \mid l^{\prime}\right\rangle, \quad \delta_{l n_{k-1}}=\left\langle l \mid n_{k-1}\right\rangle,
$$

а также введен символ Кронекера $\delta_{\left(n_{k}-n_{k-1}\right),\left(l^{\prime}-l\right)}$, который эквивалентен введению единичного множителя. Введем интегральное представление для символа Кронекера:

$$
\delta_{\left(n_{k}-n_{k-1}\right),\left(l^{\prime}-l\right)}=\int_{0}^{1} \exp \left[-2 \pi \imath\left(n_{k}-n_{k-1}-l^{\prime}+l\right) \xi_{k-1}\right] d \xi_{k-1} .
$$

Подставим (12) в (11):

$$
\begin{aligned}
\left\langle n_{k}\left|\hat{V}_{D}(\tau)\right| n_{k-1}\right\rangle=\int_{0}^{1} & \exp \left[-2 \pi \imath\left(n_{k}-n_{k-1}\right) \xi_{k-1}\right] \times \\
& \times \sum_{l^{\prime}, l} X_{l^{\prime} l}(\tau) \delta_{n_{k} l^{\prime}} \delta_{l n_{k-1}} \exp \left[2 \pi \imath\left(l^{\prime}-l\right) \xi_{k-1}\right] d \xi_{k-1} .
\end{aligned}
$$

Формулу (13) запишем в виде

$$
\begin{aligned}
\left\langle n_{k}\left|\hat{V}_{D}(\tau)\right| n_{k-1}\right\rangle=\int_{0}^{1} \exp \left[-2 \pi \imath\left(n_{k}\right.\right. & \left.\left.-n_{k-1}\right) \xi_{k-1}\right] \times \\
& \times \sum_{l^{\prime}, l} \tilde{X}_{l^{\prime} l}\left(\tau ; \xi_{k-1}\right) \delta_{n_{k} l^{\prime}} \delta_{l n_{k-1}} d \xi_{k-1},
\end{aligned}
$$

где

$$
\begin{aligned}
\tilde{X}_{l^{\prime} l}\left(\tau ; \xi_{k-1}\right)=\frac{1}{2} \hbar \Omega_{l^{\prime} l}^{R}\left(\exp \left[\imath\left(2 \pi\left(l^{\prime}-l\right) \xi_{k-1}-\left(\Omega-\omega_{l^{\prime}, l}\right) \tau\right)\right]+\right. & \\
& \left.+\exp \left[\imath\left(2 \pi\left(l^{\prime}-l\right) \xi_{k-1}+\left(\Omega+\omega_{l^{\prime}, l}\right) \tau\right)\right]\right) .
\end{aligned}
$$

Разделим сумму в (14) на две части:

$$
\left\langle n_{k}\left|\hat{V}_{D}(\tau)\right| n_{k-1}\right\rangle=\int_{0}^{1} \exp \left[-2 \pi \imath\left(n_{k}-n_{k-1}\right) \xi_{k-1}\right] \times
$$




$$
\times\left(\sum_{l^{\prime} \geqslant l} \tilde{X}_{l^{\prime} l}\left(\tau ; \xi_{k-1}\right) \delta_{n_{k} l^{\prime}} \delta_{l n_{k-1}}+\sum_{l^{\prime} \leqslant l} \tilde{X}_{l^{\prime} l}\left(\tau ; \xi_{k-1}\right) \delta_{n_{k} l^{\prime}} \delta_{l n_{k-1}}\right) d \xi_{k-1}
$$

Во второй сумме выражения (16) сделаем замену переменных $l \leftrightarrow l^{\prime}$ и учтем, что в соответствии с определением (15)

$$
\tilde{X}_{l l^{\prime}}\left(\tau ; \xi_{k-1}\right)=\tilde{X}_{l^{\prime} l}^{*}\left(\tau ; \xi_{k-1}\right)
$$

тогда после суммирования получаем

$$
\begin{aligned}
\left\langle n_{k}\left|\hat{V}_{D}(\tau)\right| n_{k-1}\right\rangle= & \int_{0}^{1} \exp \left[-2 \pi \imath\left(n_{k}-n_{k-1}\right) \xi_{k-1}\right] \times \\
& \times 2 \operatorname{Re}\left[\tilde{X}_{n_{k} n_{k-1}}\left(\tau ; \xi_{k-1}\right)\right] d \xi_{k-1} .
\end{aligned}
$$

Подставляя в выражение (17) явный вид функций $\tilde{X}_{n_{k} n_{k-1}}\left(\tau ; \xi_{k-1}\right)$ в соответствии с (15), получаем формулу (10). Заметим, что полученное выражение (10) справедливо как для $n_{k}>n_{k-1}$, так и для $n_{k}<n_{k-1}$. Для большого класса моделей $\Omega_{n_{k} n_{k}}^{R}=0$.

Подставляя данный результат в выражение (9) и используя интегральное представление для символа Кронекера

$$
\delta_{n_{k} n_{k-1}}=\int_{0}^{1} \exp \left[-2 \pi \imath\left(n_{k}-n_{k-1}\right) \xi_{k-1}\right] d \xi_{k-1}
$$

получим

$$
\begin{aligned}
& \left\langle n_{k}\left|\hat{U}_{D}\left(t_{k}, t_{k-1}\right)\right| n_{k-1}\right\rangle=\int_{0}^{1} \exp \left[-2 \pi \imath\left(n_{k}-n_{k-1}\right) \xi_{k-1}\right] \times \\
& \times\left(1-\imath \Omega_{n_{k} n_{k-1}}^{R} \int_{t_{k-1}}^{t_{k}} \cos \left(2 \pi \xi_{k-1}\left(n_{k}-n_{k-1}\right)-\left(\Omega-\omega_{n_{k} n_{k-1}}\right) \tau\right)+\right. \\
& \left.\quad+\cos \left(2 \pi \xi_{k-1}\left(n_{k}-n_{k-1}\right)+\left(\Omega+\omega_{n_{k} n_{k-1}}\right) \tau\right) d \tau\right) d \xi_{k-1}
\end{aligned}
$$

Аппроксимируя интеграл в формуле (18), получим

$$
\begin{aligned}
& \left\langle n_{k}\left|\hat{U}_{D}\left(t_{k}, t_{k-1}\right)\right| n_{k-1}\right\rangle=\int_{0}^{1} \exp \left[-2 \pi \imath\left(n_{k}-n_{k-1}\right) \xi_{k-1}\right] \times \\
& \quad \times\left[1-\imath \Omega_{n_{k} n_{k-1}}^{R}\left(\cos \left(2 \pi\left(n_{k}-n_{k-1}\right) \xi_{k-1}-\left(\Omega-\omega_{n_{k}, n_{k-1}}\right) \frac{t_{k}+t_{k-1}}{2}\right)+\right.\right. \\
& \left.\left.+\cos \left(2 \pi\left(n_{k}-n_{k-1}\right) \xi_{k-1}+\left(\Omega+\omega_{n_{k}, n_{k-1}}\right) \frac{t_{k}+t_{k-1}}{2}\right)\right)\left(t_{k}-t_{k-1}\right)\right] d \xi_{k-1} .
\end{aligned}
$$

Рассмотрим случай малых временных промежутков $\left(t_{k}-t_{k-1}\right)$, таких что

$$
\Omega_{n_{k} n_{k-1}}^{R}\left(t_{k}-t_{k-1}\right) \ll 1 .
$$

В этом случае 


$$
\begin{aligned}
& 1-\imath \Omega_{n_{k} n_{k-1}}^{R}\left(\cos \left(2 \pi\left(n_{k}-n_{k-1}\right) \xi_{k-1}-\left(\Omega-\omega_{n_{k}, n_{k-1}}\right) \frac{t_{k}+t_{k-1}}{2}\right)+\right. \\
& \left.+\cos \left(2 \pi\left(n_{k}-n_{k-1}\right) \xi_{k-1}+\left(\Omega+\omega_{n_{k}, n_{k-1}}\right) \frac{t_{k}+t_{k-1}}{2}\right)\right)\left(t_{k}-t_{k-1}\right)= \\
& =\exp \left[-\imath \Omega_{n_{k} n_{k-1}}^{R}\left(\cos \left(2 \pi\left(n_{k}-n_{k-1}\right) \xi_{k-1}-\frac{\Omega-\omega_{n_{k}, n_{k-1}}}{2}\left(t_{k}+t_{k-1}\right)\right)+\right.\right. \\
& \left.\left.+\cos \left(2 \pi\left(n_{k}-n_{k-1}\right) \xi_{k-1}+\frac{\Omega+\omega_{n_{k}, n_{k-1}}}{2}\left(t_{k}+t_{k-1}\right)\right)\right)\left(t_{k}-t_{k-1}\right)\right] d \xi_{k-1} .
\end{aligned}
$$

Учитывая (20) в выражении (19), получим ядро оператора эволюции в виде (7), где действие в энергетическом представлении определяется выражением (8), что и требовалось доказать.

Полная амплитуда перехода примет вид

$$
\begin{aligned}
\left\langle n_{f}\left|\hat{U}_{D}(t, 0)\right| n_{0}\right\rangle & = \\
& =\sum_{n_{1}, \ldots, n_{K}} \int_{0}^{1} \ldots \int_{0}^{1} \exp \left[-\imath S\left[n_{f}, n_{K}, \xi_{K} ; \ldots ;\right.\right. \\
& \left.\left.n_{k}, n_{k-1}, \xi_{k-1} ; \ldots ; n_{1}, n_{i n}, \xi_{0}\right]\right] d \xi_{0} \ldots d \xi_{K},
\end{aligned}
$$

где действие $S$ является функционалом на континууме траекторий, определяемых в дискретном пространстве переменных $n_{k}$, размеры которого определяются числом квантовых уровней исследуемой системы, и на непрерывном ограниченном на $[0,1]$ пространстве $\xi_{k}$ :

$$
S\left[n_{f}, n_{K}, \xi_{K} ; \ldots ; n_{k}, n_{k-1}, \xi_{k-1} ; \ldots ; n_{1}, n_{i n}, \xi_{0}\right]=\sum_{k=1}^{K+1} S\left[n_{k}, n_{k-1}, \xi_{k-1}\right]
$$

с условиями

$$
t_{K+1}=t, \quad n_{K+1}=n_{f}, \quad t_{0}=0 .
$$

Уравнение эволюции матрицы плотности запишем в виде

$$
\begin{aligned}
\rho_{m_{f}, n_{f}}(t)=\sum_{n_{0}, \ldots, n_{K}} \sum_{m_{0}, \ldots, m_{K}} \int_{0}^{1} \ldots \int_{0}^{1} d \xi_{0} \ldots d \xi_{K} d \zeta_{0} \ldots d \zeta_{K} \times \\
\times \rho_{n_{0}, m_{0}}(0) \exp \left[-\imath\left(S\left[n_{f}, n_{K}, \xi_{K} ; \ldots ; n_{k}, n_{k-1}, \xi_{k-1} ; \ldots ; n_{1}, n_{i n}, \xi_{0}\right]-\right.\right. \\
\left.\left.\quad-S\left[n_{f}, m_{K}, \zeta_{K} ; \ldots ; m_{k}, m_{k-1}, \zeta_{k-1} ; \ldots ; m_{1}, n_{i n}, \zeta_{0}\right]\right)\right] .
\end{aligned}
$$

Вероятность квантового перехода из чистого квантового состояния $\hat{\rho}(0)=$ $=\left|n_{i n}\right\rangle\left\langle n_{i n}\right|$ в начальный момент времени $t=0$ в конечное чистое квантовое состояние $\hat{\rho}(t)=\left|n_{f}\right\rangle\left\langle n_{f}\right|$ в конечный момент времени $t$ определяется следующим образом:

$$
P\left(n_{f}, t \mid n_{i n}, 0\right)=\sum_{n_{0}, \ldots, n_{K}} \sum_{m_{0}, \ldots, m_{K}} \int_{0}^{1} \ldots \int_{0}^{1} d \xi_{0} \ldots d \xi_{K} d \zeta_{0} \ldots d \zeta_{K} \times
$$

$\times \delta\left(n_{0}-n_{i n}\right) \delta\left(m_{0}-n_{i n}\right) \exp \left[-\imath\left(S\left[n_{f}, n_{K}, \xi_{K} ; \ldots ; n_{k}, n_{k-1}, \xi_{k-1} ; \ldots ; n_{1}, n_{i n}, \xi_{0}\right]-\right.\right.$ 


$$
\begin{aligned}
&-S {\left.\left.\left[n_{f}, m_{K}, \zeta_{K} ; \ldots ; m_{k}, m_{k-1}, \zeta_{k-1} ; \ldots ; m_{1}, n_{i n}, \zeta_{0}\right]\right)\right]=} \\
&= \sum_{n_{1}, \ldots, n_{K}} \sum_{m_{1}, \ldots, m_{K}} \int_{0}^{1} \ldots \int_{0}^{1} d \xi_{0} . . d \xi_{K} d \zeta_{0} \ldots d \zeta_{K} \times \\
& \times \exp \left[-\imath\left(S\left[n_{f}, \ldots, n_{k}, \ldots, n_{i n} ; \xi_{K}, \ldots, \xi_{k}, \ldots, \xi_{0}\right]-\right.\right. \\
&\left.\left.\quad-S\left[m_{f}, \ldots, m_{k}, \ldots, m_{i n} ; \zeta_{K}, \ldots, \zeta_{k}, \ldots, \zeta_{0}\right]\right)\right] .
\end{aligned}
$$

Разложим экспоненту по формуле Эйлера:

$$
\begin{gathered}
P\left(n_{f}, t_{f} \mid n_{i n}, t_{i n}\right)=\sum_{n_{1}, \ldots, n_{K}} \sum_{m_{1}, \ldots, m_{K}} \int_{0}^{1} \ldots \int_{0}^{1} \cos \left[S\left[n_{f}, n_{K}, \xi_{K} ; \ldots ; n_{1}, n_{i n}, \xi_{0}\right]-\right. \\
\left.-S\left[n_{f}, m_{K}, \zeta_{K} ; \ldots ; m_{1}, n_{i n}, \zeta_{0}\right]\right] d \xi_{0} \ldots d \xi_{K} d \zeta_{0} \ldots d \zeta_{K}- \\
-\imath \sum_{n_{1}, \ldots, n_{K}=1}^{N} \sum_{m_{1}, \ldots, m_{K}=1}^{N} \int_{0}^{1} \ldots \int_{0}^{1} \sin \left[S\left[n_{f}, n_{K}, \xi_{K} ; \ldots ; n_{1}, n_{i n}, \xi_{0}\right]-\right. \\
\left.-S\left[n_{f}, m_{K}, \zeta_{K} ; \ldots ; m_{1}, n_{i n}, \zeta_{0}\right]\right] d \xi_{0} \ldots d \xi_{K} d \zeta_{0} \ldots d \zeta_{K} .
\end{gathered}
$$

Учитывая, что синус - антисимметричная функция, нетрудно доказать, что мнимая часть вероятности квантового перехода равна нулю:

$$
\begin{gathered}
\sum_{n_{1}, \ldots, n_{K}} \sum_{m_{1}, \ldots, m_{K}} \int_{0}^{1} \ldots \int_{0}^{1} \sin \left[S\left[n_{f}, n_{K}, \xi_{K} ; \ldots ; n_{k}, n_{k-1}, \xi_{k-1} ; \ldots ; n_{1}, n_{i n}, \xi_{0}\right]-\right. \\
\left.-S\left[n_{f}, m_{K}, \zeta_{K} ; \ldots ; m_{k}, m_{k-1}, \zeta_{k-1} ; \ldots ; m_{1}, n_{i n}, \zeta_{0}\right]\right] \times \\
\times d \xi_{0} \ldots d \xi_{K} d \zeta_{0} \ldots d \zeta_{K}=0 .
\end{gathered}
$$

Таким образом, вероятность квантового перехода имеет вид функционального интеграла с действительным подынтегральным функционалом:

$$
\begin{aligned}
& P\left(n_{f}, t_{f} \mid n_{i n}, t_{i n}\right)=A_{\text {norm }} \sum_{n_{1}, \ldots, n_{K}} \sum_{m_{1}, \ldots, m_{K}} \int_{0}^{1} \ldots \int_{0}^{1} \times \\
& \times \cos \left[S\left[n_{f}, n_{K}, \xi_{K} ; \ldots ; n_{k}, n_{k-1}, \xi_{k-1} ; \ldots ; n_{1}, n_{i n}, \xi_{0}\right]-\right. \\
& \left.\quad-S\left[n_{f}, m_{K}, \zeta_{K} ; \ldots ; m_{k}, m_{k-1}, \zeta_{k-1} ; \ldots ; m_{1}, n_{i n}, \zeta_{0}\right]\right] d \xi_{0} \ldots d \xi_{K} d \zeta_{0} \ldots d \zeta_{K},
\end{aligned}
$$

где действие в энергетическом представлении имеет вид (8); $A_{\text {norm }}$ - константа, сохраняющая нормировочное условие

$$
\sum_{n_{f}} P\left(n_{f}, t_{f} \mid n_{i n}, t_{i n}\right)=1 .
$$

Идея о представлении вероятностей квантовых переходов интегралами по траекториям впервые была высказана Г. В. Рязановым [20]. Однако аналитическими методами провести вычисление вероятности квантовых переходов по формуле (22) не представляется возможным. Для решения подобной задачи необходимо использование методов численного интегрирования. 


\section{2. Вращательная динамика молекулы $\mathrm{N}_{2}$ под действием группы лазер-} ных импульсов. В работе [13] были опубликованы результаты экспериментов по возбуждению вращательных состояний неполярных молекул ${ }^{14} \mathrm{~N}_{2}$ и ${ }^{15} \mathrm{~N}_{2}$ последовательностью лазерных импульсов. Обсуждение полученных результатов проводилось в работах $[14,15]$.

В данных экспериментах группа молекул $\mathrm{N}_{2}$ исследовалась при температуре $T=6.3 \mathrm{~K}$, их начальное состояние определялось распределением Больцмана по энергиям вращательных уровней. Молекулы облучались группой из семи лазерных импульсов, период следования которых варьировался в интервале от 6.5 пс до 9.5 пс. Каждый лазерный импульс имел длительность порядка 500 фс. Пиковая интенсивность лазерного излучения достигала значений $I_{0}=5 \cdot 10^{12} \mathrm{~B}$ т $\mathrm{cm}^{2}$. В экспериментах наблюдалось изменение вероятностей нахождения исследуемых молекул на вращательных энергетических уровнях после действия группы импульсов. Варьировалась длительность между импульсами внутри одной группы и была исследована зависимость распределения молекул по вращательным уровням энергии в зависимости от длительности временного промежутка между лазерными импульсами.

Эксперименты с молекулами азота ${ }^{14} \mathrm{~N}_{2}$ показали, что вероятность распределения исследуемых молекул по вращательным уровням энергии существенно зависит от периода следования импульсов, как показано графиками, представленными на рис. 1, 2. Из анализа графиков следует, что при периоде следования лазерных импульсов $\tau \approx 8.38$ пс наблюдается максимальное число молекул в высоколежащих $(l \geqslant 2)$ вращательных состояниях и минимальное число молекул, находящихся на нижних энергетических уровнях $(l<2)$. Распределение вероятностей по энергиям вращательных уровней молекул азота после взаимодействия с группой ультракоротких лазерных импульсов существенно отличается от распределения Больцмана, в котором они были приготовлены в начальный момент времени. В эксперименте наблюдается явление резонанса.

Другая серия аналогичных экспериментов была проведена с двухатомными молекулами ${ }^{15} \mathrm{~N}_{2}$ (молекулами, состоящими из изотопов азота). Результаты экспериментов, то есть распределение вероятностей возбуждения энергетический уровней молекул ${ }^{15} \mathrm{~N}_{2}$ в зависимости от периода следования лазерных импульсов, представлены на рис. 2. Из анализа рис. 2 следует, что для молекулы ${ }^{15} \mathrm{~N}_{2}$ повышение вероятностей возбуждения высоколежащих вращательных состояний наблюдается при периоде следования импульсов $T \approx 8.98$ пс. Эксперименты убедительно показывают, что резонансные возбуждения молекул ${ }^{14} \mathrm{~N}_{2},{ }^{15} \mathrm{~N}_{2}$ наступают при разных периодах следования лазерных импульсов. Этот факт представляет интерес для развития технологии селективного разделения изотопов интенсивным лазерным излучением.

Дадим описание изложенных экспериментов в рамках предложенного формализма, вычисляя вероятности перехода молекулы в возбужденные состояния на основании формулы (22). В соответствии с условиями эксперимента в начальный момент времени $t=0$ вероятности наблюдения молекулы на различных уровнях подчиняется распределению Больцмана:

$$
P_{l}=\frac{1}{Z} \exp \left[-\frac{E_{l}}{k T}\right],
$$




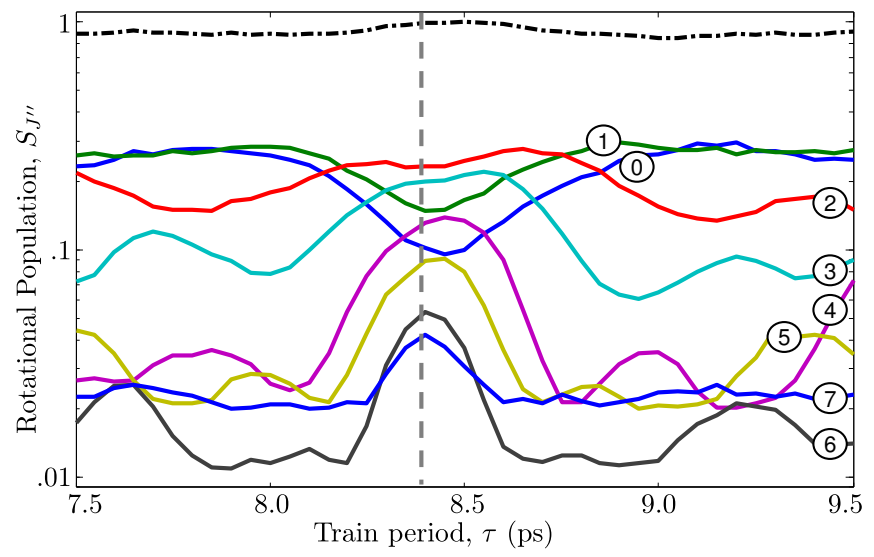

Рис. 1. (online в цвете) Зависимость вероятностей наблюдения молекул ${ }^{14} \mathrm{~N}_{2}$ на различных вращательных уровнях под действием группы лазерных импульсов с периодом $\tau$, полученных в экспериментах [13, рис. 3(a)]

[Figure 1. (color online) Rotational population of ${ }^{14} \mathrm{~N}_{2}$ molecules on different energy levels versus train period $\tau$. These experimental results are taken from [13, Fig. 3(a)] ]

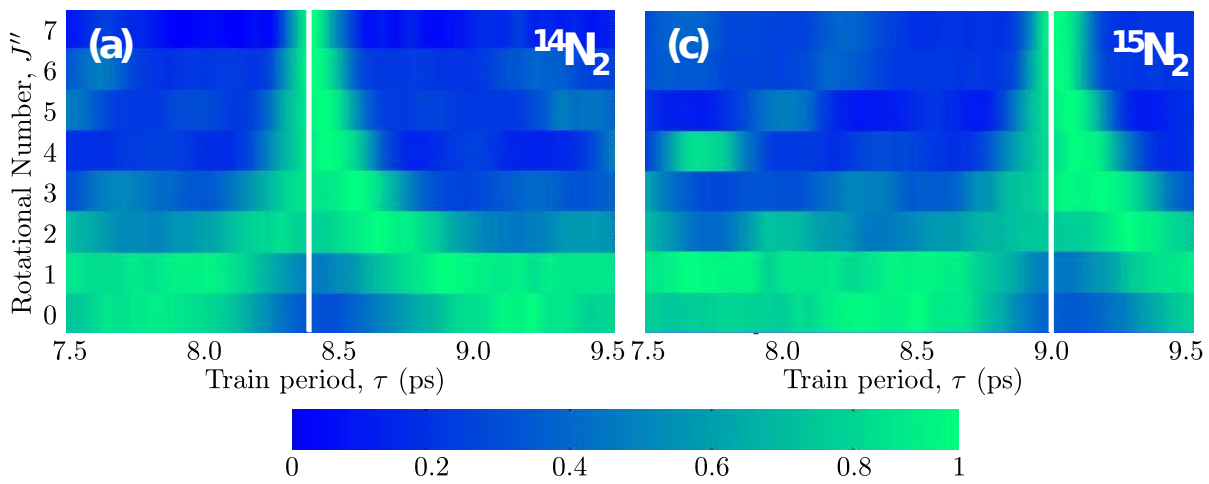

Рис. 2. (online в цвете) Распределение вероятностей наблюдения молекул ${ }^{14} \mathrm{~N}_{2}$ (левый рисунок) и ${ }^{15} \mathrm{~N}_{2}$ (правый рисунок) на различных вращательных уровнях под действием группы лазерных импульсов с периодом $\tau$, полученных в экспериментах $[13$, рис. 4(a, c)]

[Figure 2. (color online) The distribution of ${ }^{14} \mathrm{~N}_{2}$ (left) and ${ }^{15} \mathrm{~N}_{2}$ (right) probability observation with differents rotational quantum numbers versus train period. These experimental results are taken from [13, Figs. 4(a, c)]]

где

$$
Z=\sum_{l=0}^{N} \exp \left[-\frac{E_{l}}{k T}\right]
$$

- статистическая сумма; $k$ - коэффициент Больцмана; $T=6.3 \mathrm{~K}$ - абсолютная температура газа молекул; $N$ - число энергетических уровней, принятое в моделе.

Вращательные энергетические уровни молекул $E_{l}$ найдем на основании уравнения [21]

$$
-\frac{\hbar^{2}}{2 I} \frac{1}{\sin \theta} \frac{\partial}{\partial \theta}\left(\sin \theta \frac{\partial}{\partial \theta}\right) Y_{l}(\theta)=E_{l} Y_{l}(\theta)
$$


где $I=\mu R^{2}$ - момент инерции молекулы; $\mu$-приведенная масса молекулы; $R$ - расстояние между атомами; $Y_{l}(\theta)=Y_{l}^{0}(\theta, \phi), Y_{l}^{m}(\theta, \phi)$ - сферические функции $(m=0)$.

Уравнение (23) определяет вращательный энергетический спектр двухатомной молекулы

$$
E_{l}=\frac{\hbar^{2}}{2 I} l(l+1),
$$

где $l=0,1, \ldots$ - орбитальное квантовое число.

Конкретизируем взаимодействие неполярных двухатомных молекул с лазерным излучением с электрической напряженностью $E(\tau)$. В связи с тем, что дипольный момент неполярных молекул равен нулю, слагаемое, описывающее взаимодействие исследуемой молекулы с электромагнитным излучением в дипольном приближении, равно нулю. Однако под действием интенсивного лазерного излучения неполярные молекулы способны приобретать индуцированный дипольный момент, с которым впоследствии взаимодействуют. Таким образом, потенциал взаимодействия запишем в виде [13-15]

$$
\hat{V}(\tau)=-\frac{1}{4} \Delta \alpha E^{2}(\tau) \cos ^{2} \theta
$$

где $\Delta \alpha-$ постоянная, характеризующая молекулярную поляризуемость; $\theta-$ угол между осью молекулы и поляризацией лазерного излучения. Матричные элементы потенциала взаимодействия (25) -

$$
\begin{gathered}
V_{l^{\prime} l}(\tau)=-\frac{1}{4} \Delta \alpha E^{2}(\tau)\left\langle l^{\prime}\left|\cos ^{2} \theta\right| l\right\rangle \\
\left\langle l^{\prime}\left|\cos ^{2} \theta\right| l\right\rangle=2 \pi \int_{0}^{\pi} Y_{l^{\prime}}(\theta) \cos ^{2} \theta Y_{l}(\theta) d \cos \theta .
\end{gathered}
$$

Расчет матричных элементов $\left\langle l^{\prime}\left|\cos ^{2} \theta\right| l\right\rangle$ по формуле (26) проводится в данной работе численно.

Выпишем параметры молекулы ${ }^{14} \mathrm{~N}_{2}[22,23]: \Delta \alpha=1.97 \cdot 10^{-40} \mathrm{Kл} \cdot \mathrm{M}^{2} / \mathrm{B}$; $I=1.4 \cdot 10^{-46} \mathrm{\kappa г} \cdot \mathrm{M}^{2} ; \frac{\hbar^{2}}{2 I}=3.9 \cdot 10^{-23}$ Дж.

Моделировалась группа лазерных импульсов в виде эквидистантной последовательности лазерных импульсов, которые были использованы в работе [13]. Огибающая напряженности электрического поля группы лазерных импульсов принималась в виде

$$
E(\tau)=\sum_{n=-3}^{3} J_{n}(A) E_{0} \exp \left[-\frac{\left(\tau-n \tau_{\text {per }}\right)^{2}}{\tau_{\text {pul }}^{2}}\right]
$$

где $J_{n}(A)$ - функция Бесселя первого порядка; $A=2.5$ - амплитуда модуляции для создания последовательности ультракоротких лазерных импульсов; $E_{0} \approx 6 \times 10^{9} \mathrm{~B} / \mathrm{M}-$ максимальное значение огибающей напряженности электрического поля; $\tau_{\text {pul }} \approx 500$ фс-длительность каждого импульса в последовательности; 7.5 пс $\leqslant \tau_{\text {per }} \leqslant 9.5$ пс - период последовательности лазерных импульсов.

На основании формул (8), (21), (22), (24), (26), (27) с учетом значений параметров эксперимента строилась зависимость вероятностей возбуждения 
различных вращательных состояниях исследуемой молекулы ${ }^{14} \mathrm{~N}_{2}$ под действием группы лазерных импульсов при варьировании периода $\tau$ их следования.

При моделировании динамики молекул $\mathrm{N}_{2}$ мы ограничивались рассмотрением 8 вращательных уровней $(l=0,1, \ldots, 7)$. Это ограничение справедливо, так как исходя из экспериментальных данных [13] более высокие вращательные состояния имеют низкую степень возбуждения, которой можно пренебречь.

3. Результаты. При проведении исследований поведения вероятностей наблюдения молекул ${ }^{14} \mathrm{~N}_{2}$ в определенных вращательных состояниях под действием группы лазерных импульсов в зависимости от периода группы были получены результаты, представленные на рис. 3, 4.

На рис. 3, 4 представлены зависимости вероятности $P_{l}$ наблюдения молекулы ${ }^{14} \mathrm{~N}_{2}$ в различных вращательных состояниях $l$ после взаимодействия с группой лазерных импульсов с периодом $\tau$ при различных значениях максимальной величины интенсивности лазерных импульсов: $I_{0}=5 \cdot 10^{12} \mathrm{Bт} / \mathrm{cm}^{2}$ (сплошная линия), $0.5 I_{0}$ (пунктирная линия) и $2 I_{0}$ (штрихпунктирная линия). Кривая, обозначенная сплошной линией, описывает результаты проведенных экспериментов и находится с ними в согласии. На рисунках наблюдается выраженный резонанс возбуждения высоких квантовых состояний $(l \geqslant 2)$ исследуемых молекул при определенных значениях периода группы лазерных импульсов. При этом вероятности наблюдения исследуемых молекул в основном и первом возбуженном квантовом состоянии при данных значениях периода группы лазерных импульсов принимают минимальные значения. Из анализа графиков видно, что максимальная степень возбуждения молекулы ${ }^{14} \mathrm{~N}_{2}$ наблюдается при периоде импульсов $\tau \approx 8.38$ пс, что численно согласуется с результатами эксперимента. Также анализ полученных зависимостей указывает на нелинейную зависимость вероятности наблюдения исследуемых молекул от максимального значения интенсивности лазерных импульсов в группе. Из рисунков следует, что при увеличении максимальной величины интенсивности группы лазерных импульсов вероятность наблюдения молекул азота в высоких возбужденных вращательных состояниях увеличивается и при определенных интенсивностях начинает превосходить вероятность обнаружения молекулы азота в нижних квантовых состояниях.

На рис. 5 представлены распределения вероятностей наблюдения $P_{l}$ молекул в определенных вращательных состояниях $l$ при воздействии групп лазерных импульсов с периодом $\tau \approx 8.38$ пс (резонанс возбуждения молекулы ${ }^{14} N_{2}$ ) при различных интенсивностях в сравнении с начальным тепловым распределением (распределением Больцмана).

На рис. 5 сплошная линия обозначает начальное распределение молекул по вращательным квантовым состояниям - распределение Больцмана при температуре $T=6.3 \mathrm{~K}$; символы «точки»-распределение, полученное при моделировании воздействия семи импульсов с пиковой интенсивностью $I_{0}=$ $=5 \cdot 10^{12} \mathrm{~B}$ T $/ \mathrm{cm}^{2}$ (данное значение интенсивности использовалось в экспериментах [13]); «крестики» - распределение, полученное при моделировании воздействия семи импульсов с пиковой интенсивностью $I_{0}=2.5 \cdot 10^{12} \mathrm{~B}$ т $/ \mathrm{cm}^{2}$; «треугольники» - распределение, полученное при моделирования воздействия семи импульсов с максимальной интенсивностью $I_{0}=10 \cdot 10^{12} \mathrm{Bт} / \mathrm{cm}^{2}$. 


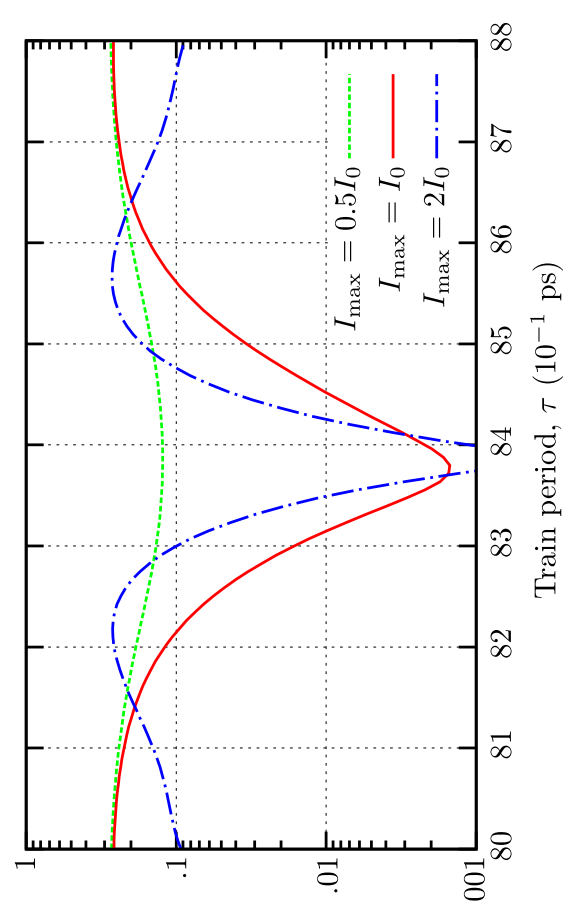

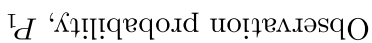

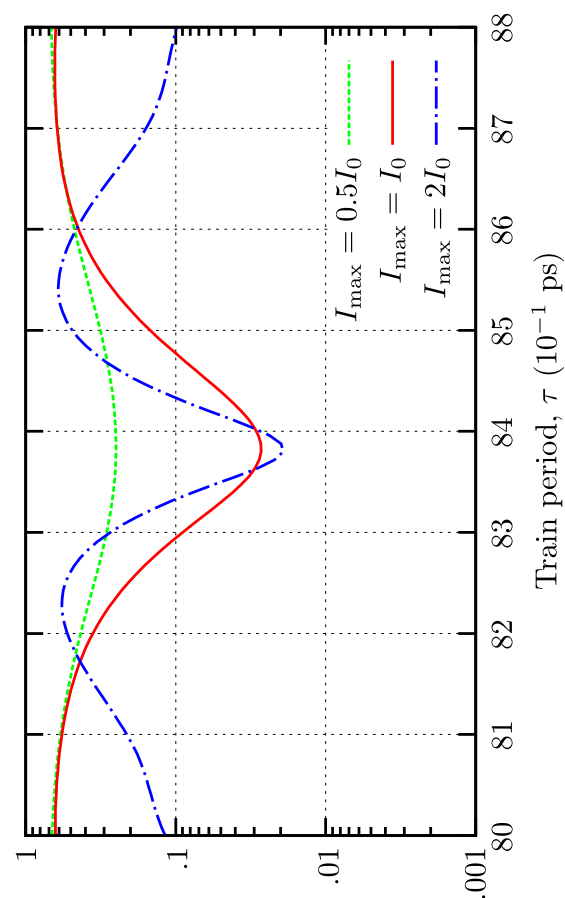

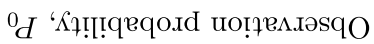

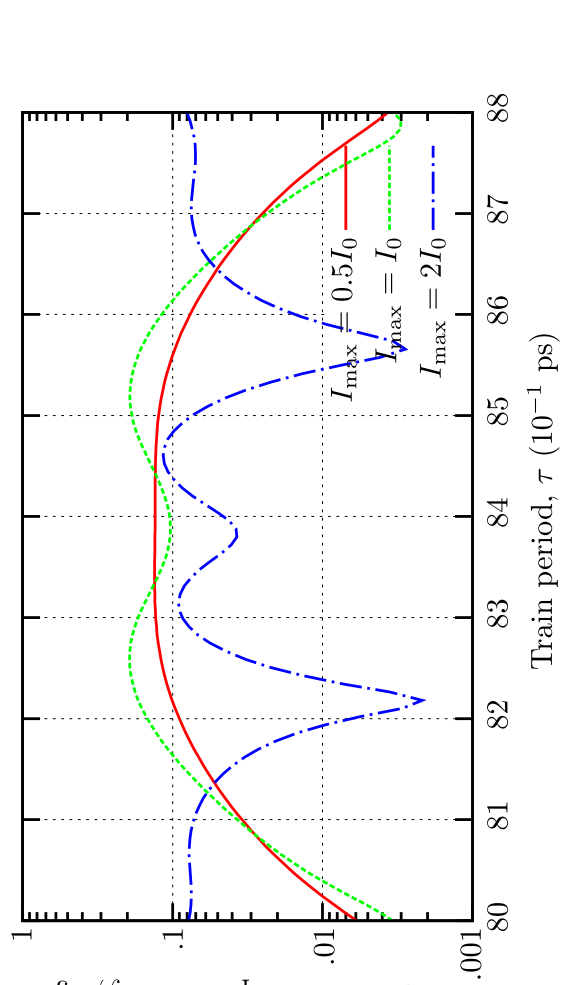

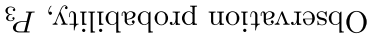

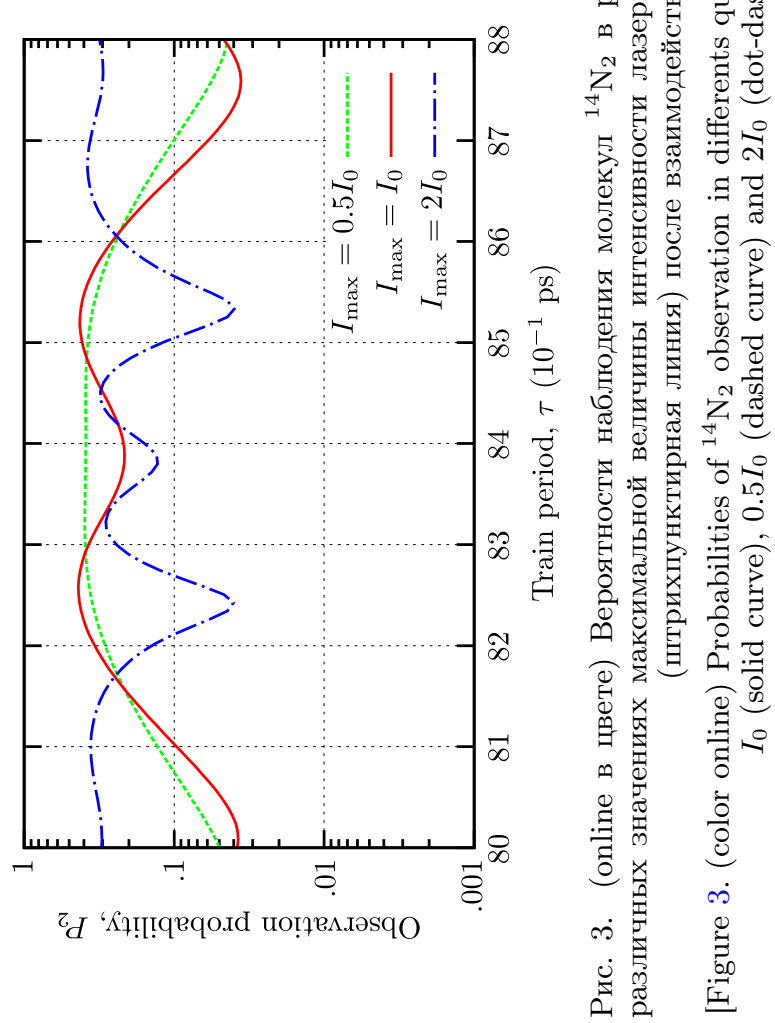

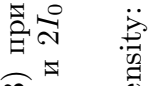

กิ

谓

0 蛋

$\| \stackrel{5}{2}$

$\sim$ 疍

药大

鞄焉

운당

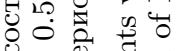

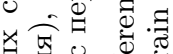

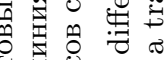

施证

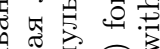

魚

范

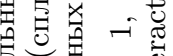

융 0

\%. क्ष 11.

宂宗

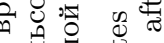

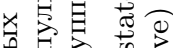

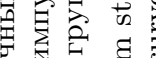

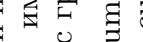

可

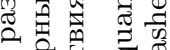

$\infty$ ल

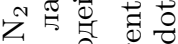

象

$\Xi \bar{\Xi}$

ㄱํㅇ

?

崩

ก 胥

$4 \frac{1}{10}$

पु 0

过

ن

두유

象

.

范

药 


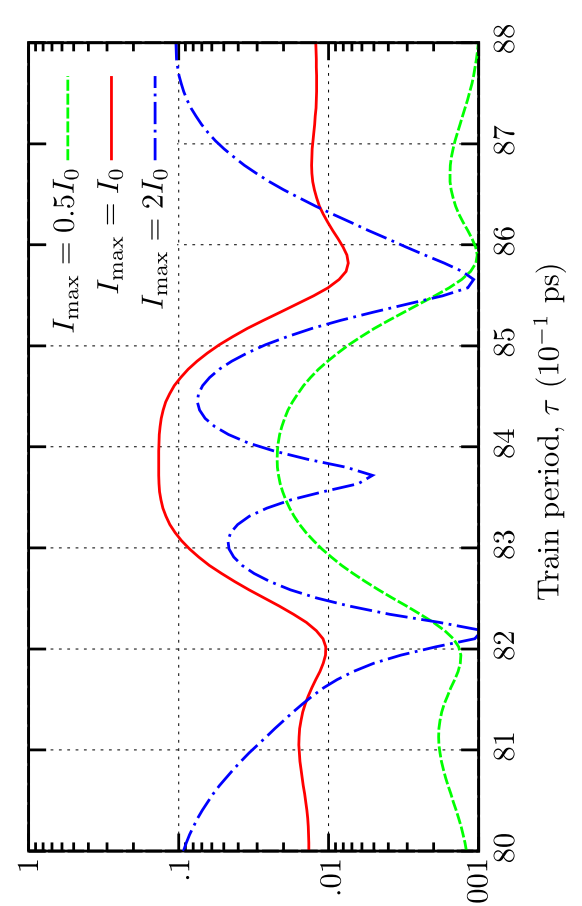

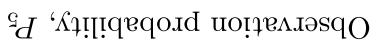

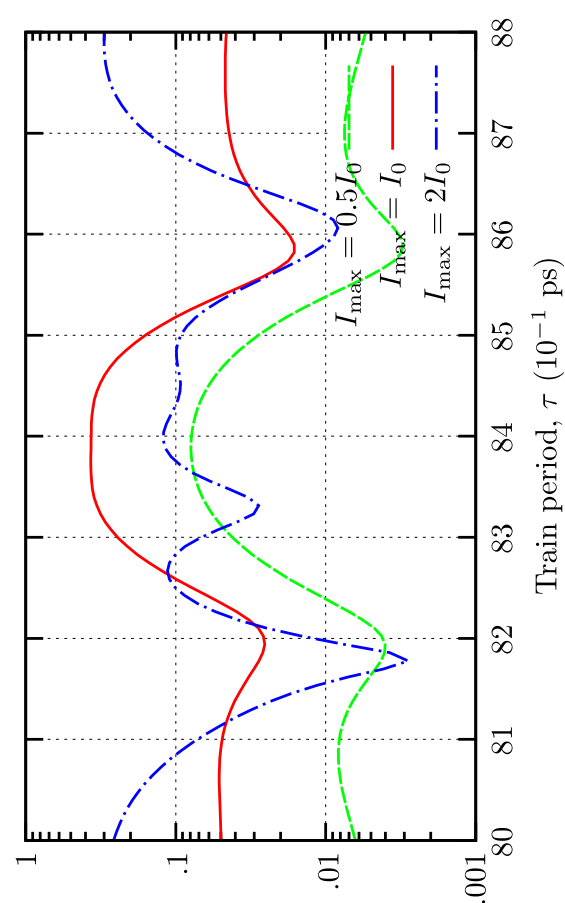

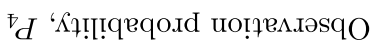

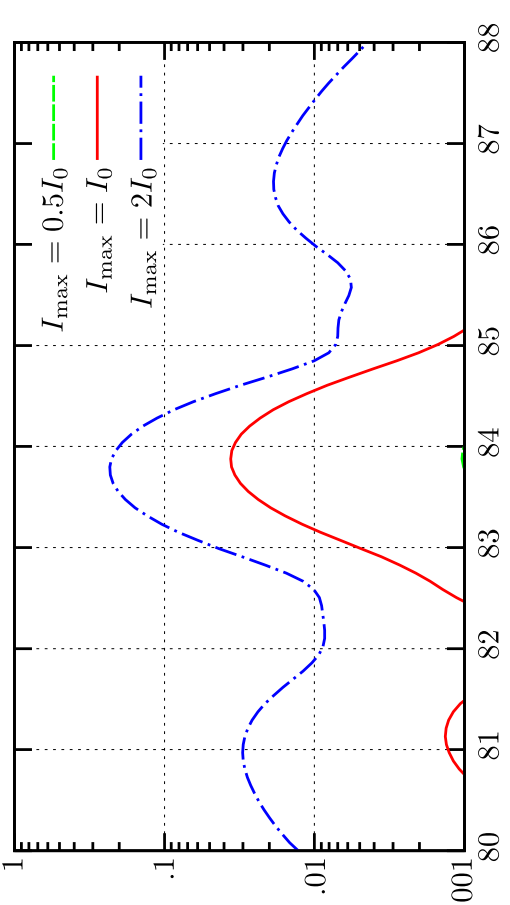

Ld '

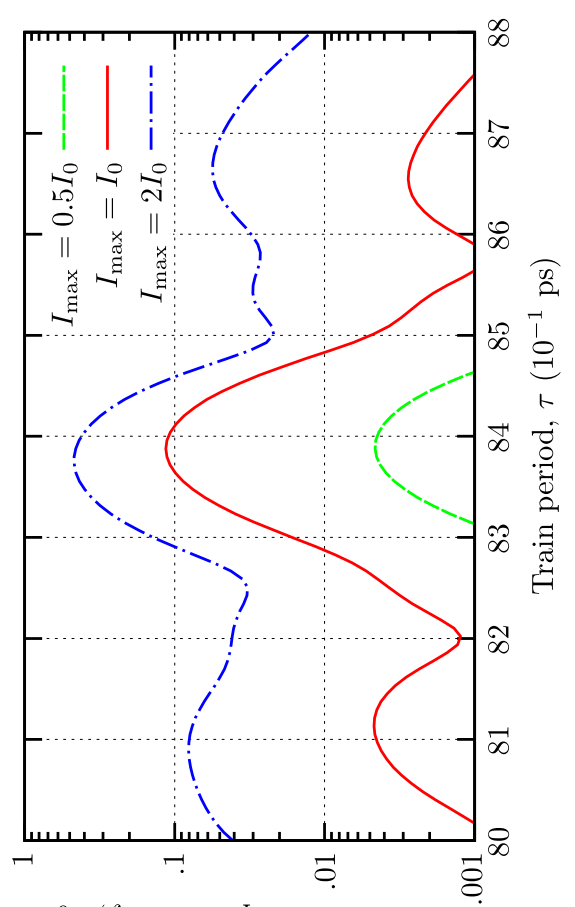

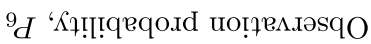

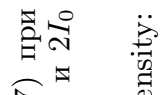

ก

15

मำ है

$\approx$

(2) 药

当突总

동요

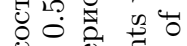

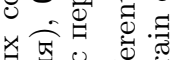

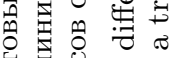

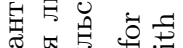

蛋芯

当目

苚

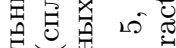

유월

丽

$\ddot{0}=$

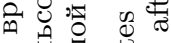

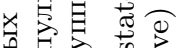

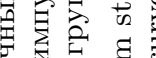

$\checkmark 0$ 的

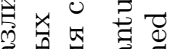

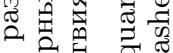

$\sim \mathrm{D}^{\circ}$

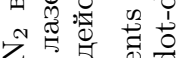

Z

穴苟出

寻

焉

응

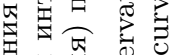

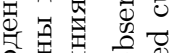

일일

蛋苛焉

क

○

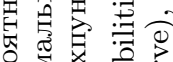

ใํํㄹ

๑ 렵 응

究四 需

4

㝵

(0) 킥 웅

$\infty$

웅

ฮี้

म葛 


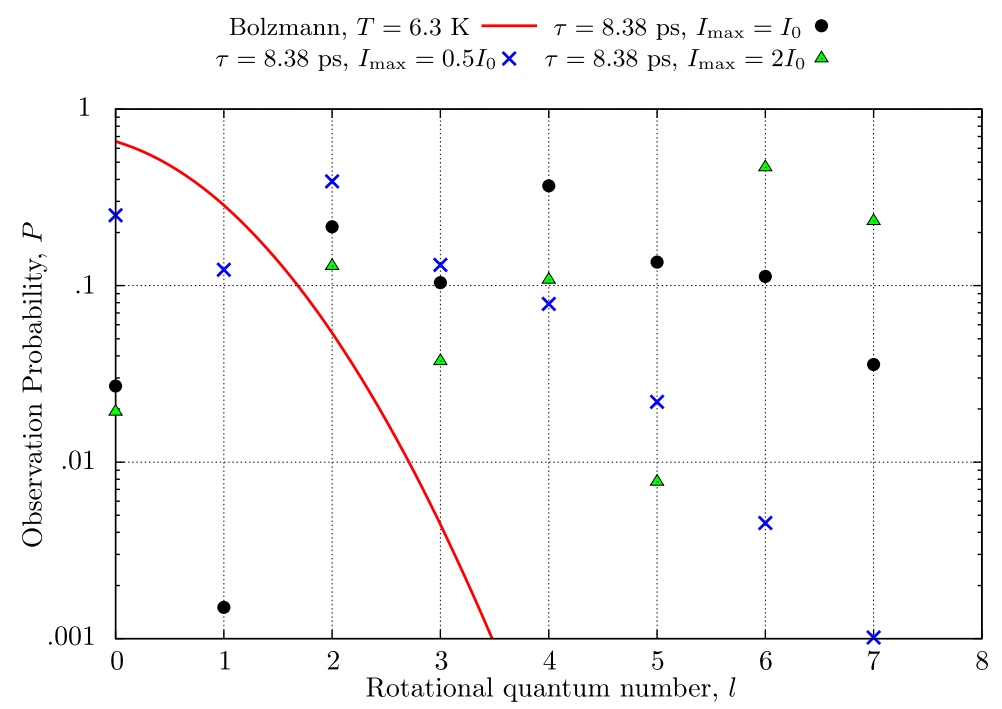

Рис. 5. (online в цвете) Распределение вероятностей наблюдения молекул ${ }^{14} \mathrm{~N}_{2}$ на различных вращательных уровнях $l$ под действием группы лазерных импульсов с периодом $\tau=8.38$ пс (резонансный случай)

[Figure 5. (color online) The distribution of ${ }^{14} \mathrm{~N}_{2}$ observation probabilities on different rotational levels $l$ after interaction with a train of laser pulses with period $\tau=8.38 \mathrm{ps}$

(resonance case)]

Из анализа графиков следует, что последовательность из семи импульсов с периодом следования $\tau=8.38$ пс возбуждает молекулы ${ }^{14} \mathrm{~N}_{2}$, которые переходят на более высокие вращательные уровни $(l \geqslant 2)$. После взаимодействия с группой ультракоротких лазерных импульсов распределение вероятностей молекул азота по вращательным состояниям не может быть сведено к распределению Больцмана при какой-либо температуре. Это указывает на нетепловой характер взаимодействия исследуемых молекул азота с полем лазерного излучения. Также наблюдается зависимость распределения вероятностей наблюдения молекул азота от интенсивности ультракоротких лазерных импульсов. При малой интенсивности (крестики) наибольшую вероятность наблюдения $(\approx 0.39)$ имеет 2-й возбужденный вращательный уровень; при интенсивности лазерного излучения, которая была использована в экспериментах [13-15], (точки) наибольшую вероятность наблюдения $(\approx 0.37)$ имеет 4-й возбужденный уровень; при вдвое большей интенсивности (треугольники) наибольшую вероятность наблюдения $(\approx 0.47)$ имеет 6-й вращательный уровень.

Проведенное моделирование представляет интерес, так как позволяет находить параметры лазерного излучения, под действием которого наблюдается заселение определенных квантовых состояний исследуемой молекулы азота ${ }^{14} \mathrm{~N}_{2}$, что может быть использовано при развитии технологий эффективного квантового когерентного контроля с высокой степень селективности.

Полученные результаты находятся в согласии с экспериментальными данными, представленными в работе [13] (см. рис. 1, 2).

Заключение. В работе предложен новый метод вычисления вероятностей переходов квантовых систем под действием электромагнитного поля излу- 
чения, вероятности переходов представлены интегралами по траекториям в пространстве энергетических состояний.

Разработанный метод позволяет проводить компьютерное моделирование динамики поведения молекулы в поле лазерных импульсов.

Предложенный метод применяется к проблеме резонансного возбуждения высоких вращательных квантовых состояний неполярных двухатомных молекул (на примере ${ }^{14} \mathrm{~N}_{2}$ ) под действием последовательности ультракоротких лазерных импульсов. Полученные в рамках численного моделирования результаты (численные значения периодов групп импульсов, при которых наблюдается квантовый резонанс) количественно согласуются с экспериментальными данными и позволяют моделировать результаты эксперимента при любых интенсивностях и периодах групп лазерных импульсов.

Благодарности. Работа выполнена при поддержке Министерства образования и науки Российской Федерации (проект 2.870.2011).

Компьютерное моделирование проводилось на суперкомпьютере «Сергей Королёв» на базе Самарского государственного аэрокосмического университета им. С. П. Королёва.

\section{ORCIDs}

Александр Александрович Бирюков: http://orcid.org/0000-0003-3955-1726

Марк Александрович Шлеенков: http://orcid.org/0000-0002-0930-4348

\section{БИБЛИОГРАФИЧЕСКИЙ СПИСОК}

1. Бирюков А. А., Шлеенков М. А. Представление вероятностей квантовых переходов функциональным интегралом в пространстве энергетических состояний / Четвертая международная конферениия «Математическая физика и ее приложения»: материалы конф.; ред. чл.-корр. РАН И. В. Волович; д.ф.-м.н., проф. В. П. Радченко. Самара: СамГТУ, 2014. С. 87.

2. Sweatlock L. A., Maier S. A., Atwater H. A., Penninkhof J. J., Polman A. Highly confined electromagnetic fields in arrays of strongly coupled Ag nanoparticles // Phys. Rev. B. vol. 71, no. 23, 235408. doi: 10.1103/physrevb.71.235408.

3. Hao E., Schatz G. C. Electromagnetic fields around silver nanoparticles and dimers // J. Chem. Phys., 2004. vol. 120, no. 1. pp. 357-369. doi: 10.1063/1.1629280.

4. Govorov A. O. Semiconductor-metal nanoparticle molecules in a magnetic field: Spinplasmon and exciton-plasmon interactions // Phys. Rev. B, 2010. vol. 82, no. 15, 155322. doi: $10.1103 /$ physrevb.82.155322.

5. Кошляков П. В., Чесноков Е. Н., Горелик С. Р., Киселев В. Г., Петров А. К. Инфракрасная многофотонная диссоциация метилтрифторсилана// Хuм. физ., 2006. Т. 25, № 6. C. $22-32$.

6. Ursrey D., Anis F., Esry B. D. Multiphoton dissociation of $\mathrm{HeH}^{+}$below the $\mathrm{He}^{+}(1 s)+$ $+\mathrm{H}(1 s)$ threshold // Phys. Rev. A. vol. 85, no. 2, 023429, arXiv: 1112.3688 [physics.atom-ph]. doi : 10.1103/physreva.85.023429.

7. Wellers Ch., Borodin A., Vasilyev S., Offenberg D., Schiller S. Resonant IR multiphoton dissociation spectroscopy of a trapped and sympathetically cooled biomolecular ion species // Phys. Chem. Chem. Phys., 2011. vol. 13, no. 42. pp. 18799-809. doi: 10.1039/ c1cp22428j.

8. Richter M., Amusia M. Ya., Bobashev S. V., Feigl T., Juranić P. N., Martins M., Sorokin A. A., Tiedtke K. Extreme Ultraviolet Laser Excites Atomic Giant Resonance // Phys. Rev. Let., 2009. vol. 102, no. 16, 163002. doi: 10.1103/PhysRevLett.102.163002.

9. Farrell J. P., Petretti S., Förster J., McFarland B. K., Spector L. S., Vanne Y. V., Decleva P., Bucksbaum P. H., Saenz A., Gühr M. Strong Field Ionization to Multiple Electronic States in Water // Phys. Rev. Let., 2011. vol. 107, no. 8, 083001, arXiv: 1103.4423 [physics.atom-ph]. doi: 10.1103/PhysRevLett.107.083001. 
10. Goodsell A., Ristroph T., Golovchenko J. A., Hau L. V. Field Ionization of Cold Atoms near the Wall of a Single Carbon Nanotube // Phys. Rev. Let., 2010. vol. 104, no. 13, 133002, arXiv: 1004.2644 [physics.atom-ph]. doi: 10.1103/PhysRevLett.104.133002.

11. Raynaud M., Kupersztych J. Ponderomotive effects in the femtosecond plasmon-assisted photoelectric effect in bulk metals: Evidence for coupling between surface and interface plasmons // Phys. Rev. B., 2007. vol.76, no.24, 241402(R). doi: 10.1103/PhysRevB.76. 241402.

12. Raynaud M., Kupersztych J. Anomalous Multiphoton Photoelectric Effect in Ultrashort Time Scales // Phys. Rev. Let., 2005. vol.95, no. 14, 147401. doi: 10.1103/PhysRevLett. 95.147401.

13. Zhdanovich S., Bloomquist C., Floß J., Averbukh I. Sh., Hepburn J. W., Milner V. Quantum Resonances in Selective Rotational Excitation of Molecules with a Sequence of Ultrashort Laser Pulses // Phys. Rev. Lett., 2012. vol. 109, no. 4, 043003, arXiv: 1201.3151 [quant-ph]. doi : 10.1103/PhysRevLett.109.043003.

14. Floß J., Fishman Shm., Averbukh I. Sh. Anderson localization in laser-kicked molecules // Phys. Rev. A, 2013. vol. 88, no. 2, 023426. doi: 10.1103/PhysRevA.88.023426.

15. Floß J., Averbukh I. Sh. Quantum resonance, Anderson localization, and selective manipulations in molecular mixtures by ultrashort laser pulse // Phys. Rev. A, 2013. vol. 86, no. 2, $021401(\mathrm{R})$, arXiv: 1110.3509 [quant-ph]. doi: 10.1103/PhysRevA.86.021401.

16. Feynman R. P., Hibbs A. R. Quantum Mechanics and Path Integrals. New York: McGrawHill, 1965.

17. Бирюков А. А., Шлеенков М. А. Вычисление вероятностей переходов квантовой системы путем интегрирования вещественных функционалов // Теоретическая физика, 2012. T. 13. C. 8-42.

18. Biryukov A., Shleenkov M. Path integral approach to the problem of rotational excitation of molecules by an ultrashort laser pulses sequence, 2014. 6 pp., arXiv: 1407.3893 [quant-ph]

19. Scully M. O., Zubairy M. S. Quantum Optics. Cambridge: Cambridge University Press, 1997. xxii+630 pp.. doi: $10.1017 /$ CB09780511813993

20. Ryazanov G. V. Quantum-mechanical probability as a sum over path // JETP, 1958. vol. 35, no. 1. pp. 121-131.

21. Ландау Л. Д., Лифшиц Е. М. Теоретическая физика. Т. 3: Квантовая механика (нерелятивистская теория). М.: Наука, 1989. 786 с.

22. Irikura K. Experimental Vibrational Zero-Point Energies: Diatomic Molecules // J. Phys. Chem. Ref. Data, 2007. vol. 36, no. 2. pp. 389-397. doi: 10.1063/1.2436891.

23. NIST Computational Chemistry Comparison and Benchmark Database/ ed. Russell D. Johnson III, Release 16a, August 2013, http://cccbdb.nist.gov.

Поступила в редакцию 19/XII/2014;

в окончательном варианте - $17 / \mathrm{II} / 2015$;

принята в печать - 08/IV/2015. 
Vestn. Samar. Gos. Techn. Un-ta. Ser. Fiz.-mat. nauki

[J. Samara State Tech. Univ., Ser. Phys. \& Math. Sci.], 2015, vol. 19, no. 2, pp. 221-240

ISSN: 2310-7081 (online), 1991-8615 (print)

doi: http://dx.doi.org/10.14498/vsgtu1392

\title{
MSC: 81Q30, 81V80
}

\section{THE QUANTUM TRANSITIONS PROBABILITY AS PATHS-INTEGRAL IN ENERGY STATES SPACE*}

\author{
A. A. Biryukov, M. A. Shleenkov \\ Samara State University, \\ 1, Academician Pavlov st., Samara, 443011, Russian Federation.
}

\begin{abstract}
By the use of the functional integration approach (paths integral approach) we present a non-perturbative method for dynamics of multi-levels quantum systems (such as atoms, molecules and nanosystems) interacting with high-intensity laser field describing. The probability of transitions between investigated quantum system states under electromagnetic field action is written as functional integral in energy representation (in investigated quantum system energy states space). In this approach we calculate probabilities of diatomic molecules transition between rotating quantum states under the ultrashort laser pulses train action by the use of numerical simulations. We investigate the dynamics of rotating quantum states population for ${ }^{14} \mathrm{~N}_{2}$ and ${ }^{15} \mathrm{~N}_{2}$ molecules interacting with a train of picoseconds laser pulses with different train period and intensity. We show for some train periods there are resonances of population transfer from low rotating quantum states of investigated molecules to high states. We study these resonances for various laser field intensities and pulses train periods. We note that in resonance case the parameters of laser field are different for ${ }^{14} \mathrm{~N}_{2}$ and ${ }^{15} \mathrm{~N}_{2}$ molecules. Obtained results indicate on the possibility of molecules rotating states selective exitation by ultrashort laser pulses train. Our numerical results are in agreement with results of experimental studies [Phys. Rev. Lett., 2012, vol. 109, 043003].
\end{abstract}

Keywords: the path integral approach, multiphoton processes, non-resonance processes, the energy representation, dinitrogen molecules, ultrashort laser pulses, non-linear interaction, rotating states selective excitation.

doi: http://dx.doi.org/10.14498/vsgtu1392

(C) 2015 Samara State Technical University.

Please cite this article in press as:

Biryukov A. A., Shleenkov M. A. The quantum transitions probability as paths-integral in energy states space, Vestn. Samar. Gos. Tekhn. Univ., Ser. Fiz.-Mat. Nauki [J. Samara State Tech. Univ., Ser. Phys. \& Math. Sci.], 2015, vol. 19, no. 2, pp. 221-240. doi: 10.14498/vsgtu1392. (In Russian)

\section{Authors Details:}

Alexander A. Biryukov (Cand. Phys. \& Math. Sci.; biryukov@samsu.ru), Head of Dept., Dept. of General \& Theoretical Physics.

Mark A. Shleenkov (Cand. Phys. \& Math. Sci.; shleenkov@list.ru; Corresponding Author), Assistant, Dept. of General \& Theoretical Physics.

${ }^{*}$ This paper is an extended version of the paper [1], presented at the Mathematical Physics and Its Applications 2014 Conference. 
Acknowledgments. This work was supported by the Ministry of Education and Science of the Russian Federation (project 2.870.2011).

Computer simulation has been performed on the supercomputer "Sergey Korolyov" at Samara State Aerospace University.

\section{ORCIDs}

Alexander A. Biryukov: http://orcid.org/0000-0003-3955-1726

Mark A. Shleenkov: http://orcid.org/0000-0002-0930-4348

\section{REFERENCES}

1. Biryukov A. A., Shleenkov M. A. The quantum transitions probability as paths-integral in energy states space, The 4 nd International Conference "Mathematical Physics and its Applications", Book of Abstracts and Conference Materials; eds. I. V. Volovich; V. P. Radchenko. Samara, Samara State Technical Univ., 2014, pp. 87 (In Russian).

2. Sweatlock L. A., Maier S. A., Atwater H. A., Penninkhof J. J., Polman A. Highly confined electromagnetic fields in arrays of strongly coupled Ag nanoparticles, Phys. Rev. B, vol. 71, no. 23, 235408. doi: 10.1103/physrevb.71.235408.

3. Hao E., Schatz G. C. Electromagnetic fields around silver nanoparticles and dimers, J. Chem. Phys., 2004, vol. 120, no. 1, pp. 357-369. doi: 10.1063/1.1629280.

4. Govorov A. O. Semiconductor-metal nanoparticle molecules in a magnetic field: Spinplasmon and exciton-plasmon interactions, Phys. Rev. B, 2010, vol.82, no. 15, 155322. doi: 10.1103/physrevb.82.155322.

5. Koshlyakov P. V., Chesnokov E. N., Gorelik S. R., Petrov A.K. Infrared multiphoton dissociation of $\mathrm{SiF}_{3} \mathrm{CH}_{3}$, Khim. Fiz., 2006, vol. 25, no. 6, pp. 22-32 (In Russian).

6. Ursrey D., Anis F., Esry B. D. Multiphoton dissociation of $\mathrm{HeH}^{+}$below the $\mathrm{He}^{+}(1 s)+$ $+\mathrm{H}(1 s)$ threshold, Phys. Rev. A, vol. 85, no. 2, 023429, arXiv: 1112.3688 [physics.atom-ph]. doi: 10.1103/physreva.85.023429.

7. Wellers Ch., Borodin A., Vasilyev S., Offenberg D., Schiller S. Resonant IR multi-photon dissociation spectroscopy of a trapped and sympathetically cooled biomolecular ion species, Phys. Chem. Chem. Phys., 2011, vol. 13, no. 42, pp. 18799-809. doi: 10.1039/c1cp22428j.

8. Richter M., Amusia M. Ya., Bobashev S. V., Feigl T., Juranić P. N., Martins M., Sorokin A. A., Tiedtke K. Extreme Ultraviolet Laser Excites Atomic Giant Resonance, Phys. Rev. Let., 2009, vol.102, no. 16, 163002. doi: 10.1103/PhysRevLett.102.163002.

9. Farrell J. P., Petretti S., Förster J., McFarland B. K., Spector L. S., Vanne Y. V., Decleva P., Bucksbaum P. H., Saenz A., Gühr M. Strong Field Ionization to Multiple Electronic States in Water, Phys. Rev. Let., 2011, vol. 107, no. 8, 083001, arXiv: 1103.4423 [physics.atom-ph]. doi: 10.1103/PhysRevLett.107.083001.

10. Goodsell A., Ristroph T., Golovchenko J. A., Hau L. V. Field Ionization of Cold Atoms near the Wall of a Single Carbon Nanotube, Phys. Rev. Let., 2010, vol. 104, no. 13, 133002, arXiv: 1004.2644 [physics.atom-ph]. doi: 10.1103/PhysRevLett.104.133002.

11. Raynaud M., Kupersztych J. Ponderomotive effects in the femtosecond plasmon-assisted photoelectric effect in bulk metals: Evidence for coupling between surface and interface plasmons, Phys. Rev. B., 2007, vol.76, no. 24, 241402(R). doi:10.1103/PhysRevB.76. 241402.

12. Raynaud M., Kupersztych J. Anomalous Multiphoton Photoelectric Effect in Ultrashort Time Scales, Phys. Rev. Let., 2005, vol. 95, no. 14, 147401. doi: 10.1103/PhysRevLett. 95. 147401.

13. Zhdanovich S., Bloomquist C., Floß J., Averbukh I. Sh., Hepburn J. W., Milner V. Quantum Resonances in Selective Rotational Excitation of Molecules with a Sequence of Ultrashort Laser Pulses, Phys. Rev. Lett., 2012, vol. 109, no. 4, 043003, arXiv: 1201.3151 [quant-ph]. doi: 10.1103/PhysRevLett.109.043003.

14. Floß J., Fishman Shm., Averbukh I. Sh. Anderson localization in laser-kicked molecules, Phys. Rev. A, 2013, vol. 88, no.2, 023426. doi: 10.1103/PhysRevA.88.023426. 
15. Floß J., Averbukh I. Sh. Quantum resonance, Anderson localization, and selective manipulations in molecular mixtures by ultrashort laser pulse, Phys. Rev. A, 2013, vol. 86, no. 2, 021401(R), arXiv: 1110.3509 [quant-ph]. doi: 10.1103/PhysRevA.86.021401.

16. Feynman R. P., Hibbs A. R. Quantum Mechanics and Path Integrals. New York, McGrawHill, 1965.

17. Biryukov A. A., Shleenkov M. A. The quantum system transitions probability as a paths integral of real functionals, Teoreticheskaia fizika, 2012, vol. 13, pp. 8-42 (In Russian).

18. Biryukov A., Shleenkov M. Path integral approach to the problem of rotational excitation of molecules by an ultrashort laser pulses sequence, 2014, 6 pp., arXiv: 1407.3893 [quant-ph]

19. Scully M. O., Zubairy M. S. Quantum Optics. Cambridge, Cambridge University Press, 1997, xxii+630 pp.. doi: 10.1017/CB09780511813993

20. Ryazanov G. V. Quantum-mechanical probability as a sum over path, JETP, 1958, vol. 35, no. 1 , pp. 121-131.

21. Landau L. D., Lifshitz E. M. Course of Theoretical Physics, vol. 3, Quantum mechanics. Non-relativistic theory. London, New York, Paris, Los Angeles, Pergamon Press Ltd., 1958, xii+515 pp.

22. Irikura K. Experimental Vibrational Zero-Point Energies: Diatomic Molecules, J. Phys. Chem. Ref. Data, 2007, vol. 36, no. 2, pp. 389-397. doi: 10.1063/1.2436891.

23. NIST Computational Chemistry Comparison and Benchmark Database, ed. Russell D. Johnson III, Release 16a, August 2013, http://cccbdb.nist.gov.

Received 19/XII/2014; received in revised form $17 / \mathrm{II} / 2015$; accepted 08/IV/2015. 